\title{
ANN based Load Prediction in Residential Buildings
}

\author{
BIBIN G S1, CHINCHU M2 \\ Assistant Engineer, KSEB, Kerala, India ${ }^{1}$ \\ Assistant Professor, Electronics \& communication, College of Engineering Kidangoor, Kerala, India ${ }^{2}$
}

\begin{abstract}
Load prediction of residential buildings is a major area in which research has been going on.The primary aim is to improve the efficiency of demand prediction models. The conventional systems using fixed set point based products like SCADA is not seen to be giving the desired results. In such models the large gap between actual and predicted values of energy consumption causes huge error and it makes such systems unsuitable for use. In such a situation the use of thermal energy model with ANN(artificial neural network)is found to be giving better results with less gap in energy demand and prediction. This project is aimed to study the energy consumption pattern of residential buildings in kerala. Few physical parameters are considered in developing the the building model
\end{abstract}

Keywords: Load Prediction, SCADA, ANN, TOD, DISCOM.

\section{INTRODUCTION}

This project focus mainly on the energy consumption of residential buildings. The demand always goes on increasing day by day .There are many interdependent systems which make the building a complex system to predict the energy consumption .The supplied Energy can either be deficit or surplus. Making energy demand and supply gap to the negligible extend is our greatest challenge. Different types of control systems were used to handle these tasks, such control systems acquire data and then process it to control the parameters so as to obtain the desired output. SCADA is one among those commercially successful product handling the required tasks. SCADA based systems uses predefined static schedules and set points. These schedules/set-points in SCADA are limited. It makes such a system unable to cater the continuously changing parameters of energy demand

\section{OBJECTIVE}

The objective of this project are

1. Energy forecasting using artificial neural network

2. Economical and reliable operation of distribution network

3. Profitable to customer and company

4. Develop energy strategies for government

\section{METERING SYSTEM ARCHITECTURE}

Metering system architecture is the conceptual model that defines the structure of the metering system; it describes and represents the structure of the metering system components, how the components are organized and how they interact. There are many different types of metering equipment, each with a host of options and variations. The purpose of this section is to discuss the overall architecture of a comprehensive metering system from the base sensors to the user interface. Understanding the metering system architecture will help in the overall planning process, especially when planning for the development of enterprise-level systems.

A. Metering Device: The metering device consists of four basic elements: sensors (A), signal transmission (B), flow computer (C), and internal data storage (D). Every meter starts with sensor technology. Electric meters require sensors for voltage and current. Other meters rely on sensors for frequency, pressure, velocity, temperature or other parameters. The sensors then must transfer a signal (B) to a processor or computer (C). The sensor signal (B) may be wired or wireless, depending on the device. The processor, or flow computer (C), calculates the values of interest for example, kilowatts per hour, pounds, and gallons per minute. The meter may also have some type of internal memory (D). In the case of an electric smart meter, the internal memory may be capable of storing months of high-frequency interval energy data in addition to a host of additional sensor data. In the case of a basic steam meter, internal memory may be limited to dynamic 


\section{International Journal of Advanced Research in Computer and Communication Engineering}

Vol. 10, Issue 5, May 2021

\section{DOI 10.17148/IJARCCE.2021.105126}

short-term storage of sensor data being processed by the flow computer. Some meters, such as your basic water meter, may have no internal storage memory.

B. Communications: In this metering system component, data are communicated (E) from the metering device to a data acquisition system. In practice, this component is actually two elements. As illustrated in Figure A, the meter transmits the data and the data acquisition system (F) receives the data. Originally, this process involved someone manually reading the meter, taking notes, and later writing or typing the noted meter readings as the data moved into the next component. While manually connecting and downloading metered data is still in practice today, the state of the technology is moving more towards remote access and automation. The communications process may be limited to one-way communications, for example, automatic meter reading [AMR] systems, or may be capable of two-way communications, for example, advanced metering infrastructure [AMI] systems. The communications process may be wired or wireless; the signal type may be analog, pulse, or digital; the data may be communicated using any number of protocols; and data may follow any number of pathways to get to the next component. Although automated communications and remote access reduce labor costs and transcribing errors, this component can still be the weak link in the overall metering system. This communications element is one of the most critical in the entire system architecture. Many metering system data losses can be attributed to a failure in this component.

C. Data Acquisition: For facilities with multiple meters, the data acquisition system (F) scans, reads, and records the meter data. If the meter's flow computer did not apply a timestamp to the data package, the data acquisition system should apply a timestamp as the meter data are recorded. If multiple systems are responsible for applying the timestamp to the metered data, it is important that the time clocks be synchronized with a process for checking time clock accuracy and re synchronizing periodically. The data acquisition system is frequently an interim component between the meter(s) and the central data storage $(\mathrm{H})$. But for small and local systems, the data acquisition system may include the local central data storage function. For facilities that use a building automation system (BAS) to read and record metered data, the BAS may serve as the data acquisition system. A central energy information system (EIS) computer may serve this purpose. For larger metering systems, the data acquisition system serves as an intermediate step; collecting data from nearby meters and transmitting the data onto the next element. Because it takes time to scan, read, transfer, and record metered data, this component can be a weak link in the process. Trying to transfer too much data in too little time or connecting too many meters to a single data acquisition system can result in skipped scans and lost data. For very large metering systems, multiple data acquisition systems may be warranted.

D. Site-Level User Interface: The site-level user interface $(\mathrm{K})$ is the component where the data are available for data analysis and data can be visualized using charts or dashboards. The user interface can access a copy of the pre-processed data $(\mathrm{N})$ and may even have direct access to the metering device's internal data storage (D) and the flow computer (C). In this illustration, the user interface is for a local, site-level, facility user. In the case of an enterprise-level metering system, three more components may exist above the site-level system.

E. External Communications: Data from the facility data storage can be further communicated (L) to an enterprise level. In this communication process, a copy of the raw data $(\mathrm{H})$, the per-processed data $(\mathrm{J})$, or both may be communicated to the enterprise level. The transmission process may use Ethernet, cellular, hard-wired telephone, or even satellite communications. Poor communications in this step does not necessarily mean permanently lost data because data transfer can always be validated and re transmitted if necessary.

\section{NEURAL NETWORKS}

Neural networks are composed of simple elements operating in parallel. These elements are inspired by biological nervous systems.

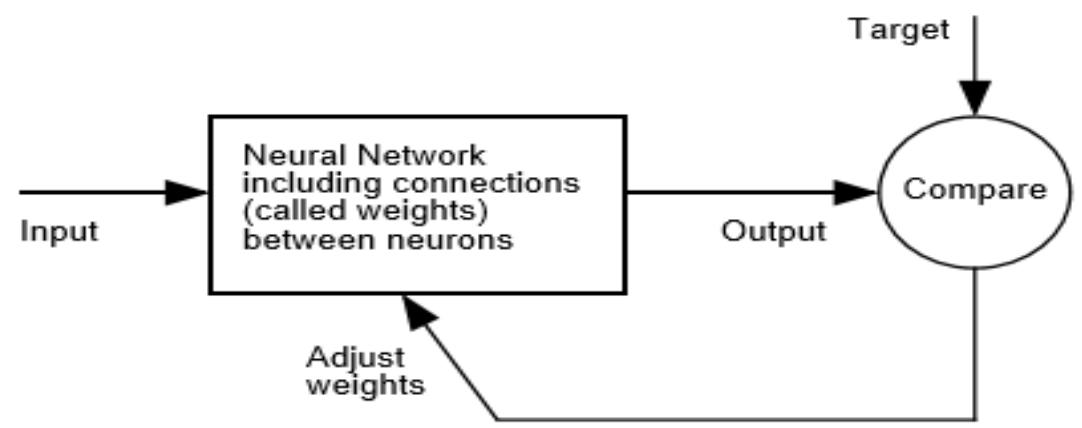

Fig: I A Neural network 
As in nature, the network function is determined largely by the connections between elements. We can train a neural network to perform a particular function by adjusting the values of the connections (weights) between elements. Commonly neural networks are adjusted, or trained, so that a particular input leads to a specific target output. Such a situation is shown below. There, the network is adjusted, based on a comparison of the output \& the target, until the network output matches the target. Typically many such input/target pairs are used, in this supervised learning, to train a network.

\section{SELECTION OF ANN}

The major stages in ANN model development are:

1.Create and configure the network

2.Train the network

3.Use the network

Feed forward back propagation Type ANN is selected because it is easy to converge and best suited for these type of applications.Matlab program was used to train and test the neural network and observe the results. Many number o training algorithms are available in Artificial Neural Networks.Here back propagation algorithm is used to train the network.

\section{LEARNING TECHNIQUES USED IN NEURAL NETWORKS}

A. Supervised Learning: In supervised learning, the training data is input to the network, and the desired output is known, weights are adjusted until production yields desired value.

B. Unsupervised Learning: The input data is used to train the network whose output is known. The network classifies the input data and adjusts the weight by feature extraction in input data.

C. Reinforcement Learning: Here the value of the output is unknown, but the network provides the feedback whether the output is right or wrong.

D. Offline Learning: The adjustment of the weight vector and threshold is made only after all the training set is presented to the network. It is also called Batch Learning.

E. Online Learning: The adjustment of the weight and threshold is made after presenting each training sample to the network.

\section{LEARNING DATA SETS IN NEURAL NETWORKS}

A. Training Set: A set of examples used for learning, that is to fit the parameters of the network.One Epoch comprises of one full training cycle on the training set.

B. Validation Set: A set of examples used to tune the parameters of the network. For example to choose the number of hidden units in a Neural Network.

C. Test Set: A set of examples used only to assess the performance of a fully specified network or to apply successfully in predicting output whose input is known

\section{WORK METHODOLOGY}

The project is planned to be carried out in three stages. The different works of these stages are listed as follows

\section{A. Stage-1}

The project starts from a physical model of a building The parameters like sq $\mathrm{ft}$ area, connected load, temperature are taken .The type of loads are of greater concern.All these parameters are chosen as the control variables A system engineering approach requires a clear definition of the optimization objectives, while all available control variables and their ranges have to be considered. The data (control variables and consumption pattern) has to be collected on all weather seasons. The data collection is the most important stage of this process. Then this data has to be pre-processed to analyse sensitive variables. A statistical approach is required at this stage.

\section{B.Stage-2}

In this stage ANN is used to learn the energy demand pattern. A well trained ANN is able to create the relationship between inputs. A multilayer perceptron is most suitable. MATLAB is used for simulation purposes.

\section{Stage-3}

This stage is the formulation of the output or the predicted data from the results of trained ANN. An user interface is developed in MATLAB GUIDE which gives the facility for easy graphical user interface for non technical personnel for the use of the system.In this user interface if we give the input parameters the predicted consumption will be displayed. 


\section{International Journal of Advanced Research in Computer and Communication Engineering}

Vol. 10, Issue 5, May 2021

DOI 10.17148/IJARCCE.2021.105126

The data collected were used for the general prediction as well as the data obtained on a TOD basis was used for half hour demand prediction.

\section{DATA COLLECTION}

The consumer data is collected from DISCOM data.The essential details of the consumer like the connected load, area of the consumer premises, the average consumption in a monthly pattern for the whole year has been collected.

\section{RESULTS}
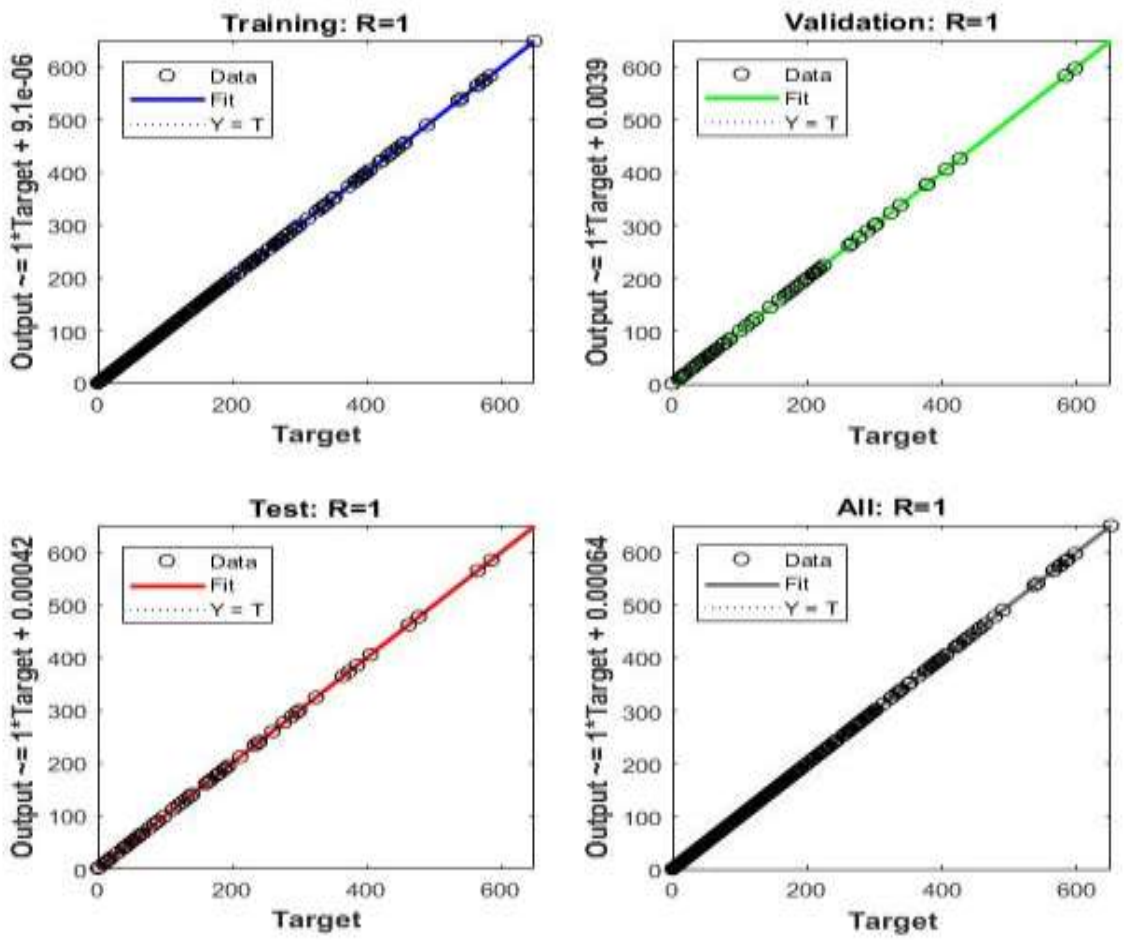

Fig II: Training results of ANN

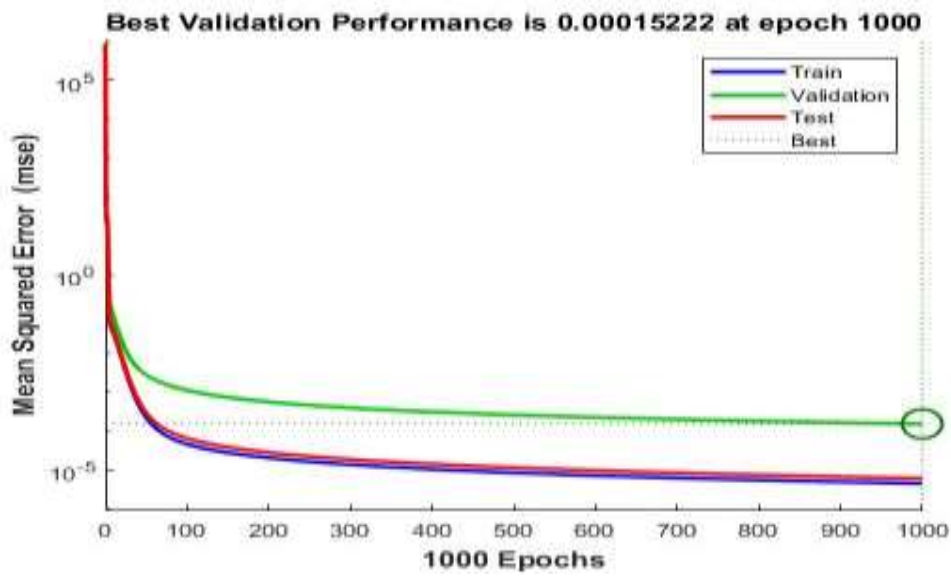

Fig: III Training perormance of ANN

The next major step is the normalization of the input data. As different input variables with different data ranges it is essential for the normalization to attain a converged result.

\section{NORMALIZATION}

Different quantities like Energy ,connected load etc are trained with neural network.As the magnitude of input data vector changes the prediction will be biased.It means the high magnitude data have more control over prediction Different 


\section{International Journal of Advanced Research in Computer and Communication Engineering}

Vol. 10, Issue 5, May 2021

DOI 10.17148/IJARCCE.2021.105126

quantities with different ranges take more converging time.So data vector normalization is required.The input database was modified by preprocessing by making all value into a normal range of zero and one each input variable $\mathrm{x}$ was hence normalized to $\mathrm{x}(\mathrm{n})$ by using

$$
\mathrm{X}(\mathrm{n})=0.8(\mathrm{x}-\mathrm{x} \min ) / \mathrm{x} \max -\mathrm{x} \min +0.1
$$

\section{USER INTERFACE DESIGN}

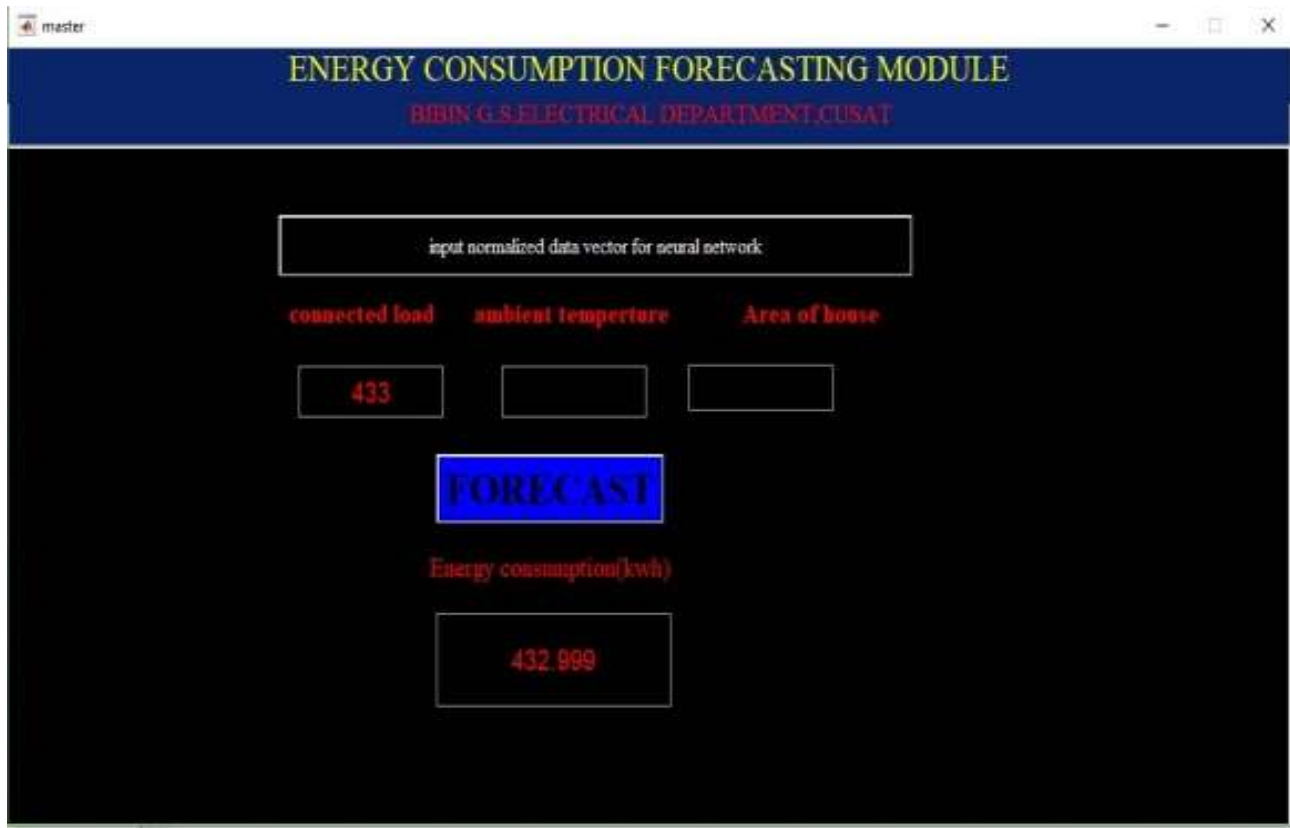

Fig: IV User Interface

A prototype user interface was developed for forecasting the energy with trained neural network.It take the input parameters into the trained neural network and forecasts the load.

\section{CONCLUSION}

The ANN is trained with the data collected from the Discoms as well as from the TOD meters. The best fit of the ANN is chosen. A Graphical user interface is developed for the simplicity of the user. for the inputting the parameters. Finally we were able to predict the average consumption of a particular consumer as well as the half hour load pattern of a consumer.

\section{ACKNOWLEDGMENTS}

The authors gratefully acknowledge, consumers for providing the data for carrying out this work. Also thank and grateful towards College of Engineering Kidangoor's Department of Electronics and Communication for their valuable effective timely support throughout the design.

\section{REFERENCES}

[1]. An ANN-GA Semantic Rule-Based System to Reduce the Gap Between Predicted and Actual Energy Consumption in Buildings Baris Yuce, Member, IEEE, and Yacine Rezgui, IEEE Transaction- 10.1109/TASE.2015.2490141

[2]. A. Balaras, K. Droutsa, E. Dascalaki, and S. Kontoyiannidis, "Heating energy consumption and resulting environmental impact of European apartment buildings," Energy Build., vol. 37, no. 5, pp. 429-442, Aug. 2004.

[3]. L. Perez-Lombard, J. Ortiz, and C. Pout, “A review on building energy consumption information," Energy Build., vol. 40, no. 3, pp. 394-398, Mar. 2007.

[4]. "Directive 2002/91/EC of the European parliament and of the council of 16 December 2002 on the energy performance of buildings," Official J. Eur. Communities, pp. L 65-71, Dec. 2010. [4] B. Yuce, H. Li, Y. Rezgui, I. Petri, B. Jayan, and C. Yang, "Utilizing artificial neural network to predict energy consumption and thermal comfort level: An indoor swimming pool case study," Energy Build., vol. 80, pp. 45-56, Sep. 2014.

[5]. 1 .Forecasting Electricity Consumption with Neural Networks and Support Vector Regression- Omer F. Demirelb, Selim Zaimc, Social and Behavioral Sciences 58 ( 2012 ) $1576-1585$

[6]. Energy Forecasting using Artificial Neural Network G.Tamizharasi, S.Kathiresan, K.S.Sreenivasan, Ijareeie Vol.3, Iss3, Mar 2014 pp75 68-76

[7]. Artificial neural networks in energy applications in buildings Soteris A. Kalogirou, ijlct ,article-abstract 1/3/201/743439 pp 201-16

[8]. Development of Neural Network Prediction Model of Energy Consumption, Maryam Jamela Ismail, Rosdiazli Ibrahim, Idris Ismail, World Academy of Science, Engineering and Technology, International Journal of Energy and Power Engineering Vol:5, No:10, 2011 pp 1367-72 